\title{
Stability Analysis of the Reproduction Operator in Bacterial foraging Optimization
}

\author{
Arijit Biswas \\ Jadavpur University \\ Kolkata, India
}

\author{
Swagatam Das \\ Jadavpur University, \\ Kolkata, India
}

\author{
Ajith Abraham \\ Q2S, NTNU \\ Trondheim, Norway \\ ajith.abraham@ieee.org
}

\author{
Sambarta Dasgupta \\ Jadavpur University \\ Kolkata, India
}

\begin{abstract}
One of the major operators of Bacterial Foraging Optimization Algorithm (BFOA) is the reproduction phenomenon of virtual bacteria each of which models one trial solution of the optimization problem. During reproduction, the least healthier bacteria (with a lower accumulated value of the objective function in one chemotactic lifetime) die and the other healthier bacteria each split into two, which then starts exploring the search place from the same location. Thus the population size is maintained constant in BFOA. In this paper we try to model the reproduction dynamics and then analyzed the stability of the reproductive system very near to equilibrium. We also find the relative positions of two bacteria for which a stable reproduction event can take place in a one dimensional fitness landscape.
\end{abstract}

Categories and Subject Descriptors

I.2.8 [Artificial Intelligence]: Problem Solving, Control Methods, and Search --- Heuristic methods; G.1.6 [Numerical Analysis]: Optimization --- Global optimization; G.3 --- Probabilistic algorithms

\section{General Terms}

Algorithms

\section{Keywords}

Bacterial foraging, computational chemotaxis, stability analysis

\section{INTRODUCTION}

To tackle several complex search problems of real world, scientists have been looking into the nature for years - both as model and as metaphor - for inspiration.

Permission to make digital or hard copies of all or part of this work for personal or classroom use is granted without fee provided that copies are not made or distributed for profit or commercial advantage and that copies bear this notice and the full citation on the first page. To copy otherwise, or republish, to post on servers or to redistribute to lists, requires prior specific permission and/or a fee.

CSTST 2008, October 27-31, 2008, Cergy-Pontoise, France.

Copyright 2008 ACM 978-1-60558-046-3/08/0003.\$5.00.
Optimization is at the heart of many natural processes like Darwinian evolution, group behavior of social insects and the foraging strategy of other microbial creatures. Natural selection tends to eliminate species with poor foraging strategies and favor the propagation of genes of species with successful foraging behavior, as they are more likely to enjoy reproductive success.

Since a foraging organism or animal takes necessary action to maximize the energy utilized per unit time spent for foraging, considering all the constraints presented by its own physiology such as sensing and cognitive capabilities, environment (e.g. density of prey, risks from predators, physical characteristics of the search space), the natural foraging strategy can lead to optimization and essentially this idea can be applied to real-world optimization problems. Based on this conception, Passino proposed an optimization technique known as Bacterial Foraging Optimization Algorithm (BFOA) [1, 2, and 3]. Until date, the algorithm has successfully been applied to real world problems like optimal controller design [1,2], harmonic estimation [4], transmission loss reduction [5], pattern recognition [6], controller synthesis for active power filters [7] and, power system optimization [8]. BFOA is a newly added member in the coveted realm of Swarm Intelligence [9, 10, 16, 17], which also includes powerful optimization techniques like the Particle Swarm Optimization (PSO) [10, 11] and Ant Colony Optimization (ACO) [12].

One of the major steps of BFOA is the event of reproduction in which the bacterial population is at first sorted in the order of ascending accumulated cost (value of the objective function to be optimized), then the worst half of the population containing least healthy bacteria is liquidated while all the members of the better half is split into two bacteria, which start exploring the search space from the same location on the fitness landscape. As pointed out by Passino, this phenomenon finds analogy with the elitistselection mechanism of the classical evolutionary algorithms (EA) [1, 2, and 13]. Bacteria in the most favorable environment (i.e., near an optima) gain a selective advantage for reproduction through the cumulative cost.

This paper provides a simple mathematical analysis of the reproduction mechanism in BFOA. We focus our attention on a simple two-bacterial system working over a one dimensional fitness landscape and try to form the reproduction event as dynamics [14]. The resultant dynamics is then represented in a state space, where the displacement and velocity of a bacterium are assumed to be the state variables. Then we undertake a stability analysis of reproduction very near to the equilibrium point and try to 
find the relative positions of the two bacteria for which only a stable reproduction event can take place.

The rest of the paper is organized as follows. Section 2 provides a brief description of the bacterial foraging optimization algorithm. In Section 3 we show how we can model reproduction mathematically. Then the stability analysis of the obtained dynamics is carried out in Section 4. Finally the conclusions are drawn with a discussion on the future research scopes in Section 5.

\section{BACTERIAL FORAGING OPTIMIZATION ALGORITHM}

The bacterial swarm proceeds through four principal mechanisms namely chemotaxis, swarming, reproduction and elimination-dispersal [1]. Below we briefly describe each of these processes and finally provide a pseudo-code of the entire algorithm.

I. Chemotaxis: This process simulates the movement of an E.coli cell through swimming and tumbling via flagella. Biologically an E.coli bacterium can move in two different ways. It can swim for a period of time in the same direction or it may tumble, and alternate between these two modes of operation for the entire lifetime. Suppose $\theta^{i}(j, k, l)$ represents $i$-th bacterium at $j$-th chemotactic, $k$-th reproductive and 1-th elimination dispersal step. $\mathrm{C}(\mathrm{i})$ is the size of the step taken in the random direction specified by the tumble (run length unit). Then in computational chemotaxis the movement of the bacterium may be represented by

$$
\theta^{i}(j+1, k, l)=\theta^{i}(j, k, l)+C(i) \frac{\Delta(i)}{\sqrt{\Delta^{T}(i) \Delta(i)}}
$$

Where $\Delta$ indicates a unit length vector in the random direction.

II. Swarming: An interesting group behavior has been observed for several motile species of bacteria including E.coli and S. typhimurium, where stable spatio-temporal patterns (swarms) are formed in semisolid nutrient medium. A group of E.coli cells arrange themselves in a traveling ring by moving up the nutrient gradient when placed amidst a semisolid matrix with a single nutrient chemo-effecter. The cells when stimulated by high level of succinate release an attractant aspertate, which helps them to aggregate into groups and thus move as concentric patterns of swarms of high bacterial density. The cell to cell, signaling in E.coli swarm may be represented with the following function.

$$
\begin{aligned}
& J_{c c}\left(\theta^{i}(j, k, l)\right)=\sum_{i=1}^{s}\left[-d_{\text {attractant }} \exp \left(-w_{\text {attractant }} \sum_{m=1}^{p}\left(\theta_{m}-\theta_{m}^{i}\right)^{2}\right)\right]+ \\
& \sum_{i=1}^{s}\left[h_{\text {repellant }} \exp \left(-w_{\text {repellant }} \sum_{m=1}^{p}\left(\theta_{m}-\theta_{m}^{i}\right)^{2}\right)\right]
\end{aligned}
$$

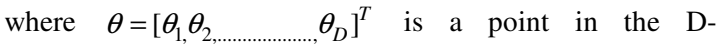

dimensional search domain.

III. Reproduction: The least healthy bacteria eventually die while each of the healthier bacteria (those yielding lower value of the cost function) asexually split into two bacteria which are placed at the same location of the fitness landscape. This keeps the swarm size constant.

IV. Elimination and Dispersal: Gradual or sudden changes in the local environment where a bacterium population lives may occur due to various reasons e.g. a significant local rise of temperature may kill a group of bacteria that are currently in a region with a high concentration of nutrient gradients. Events can take place in such a fashion that all the bacteria in a region are killed or a group is dispersed into a new location. To simulate this phenomenon in BFOA some bacteria are liquidated at random with a very small probability while the new replacements are randomly initialized over the search space.

\section{ANALYSIS OF THE REPRODUCTION STEP IN BFOA}

Let us consider a small population of two bacteria that sequentially undergoes the four basic steps of BFOA over a one-dimensional objective function. The bacteria lives in continuous time and at the $t$-th instant its position is given by $\theta(t)$. Below we list a few assumptions that were considered for the sake of gaining mathematical insight.

\subsection{Assumptions}

i) The objective function $\mathbf{J}(\theta)$ is continuous and differentiable at all points in the search space.

ii) The analysis applies to the regions of the fitness landscape where gradients of the function are small i.e., near to the optima. The region of fitness landscapes between $\theta_{1}$ and $\theta_{2}$ is monotonous at the time of reproduction.

iii) During reproduction, two bacteria remain close to each other and one of them must not superpose on another (i.e. $\left|\theta_{2}-\theta_{1}\right| \rightarrow 0$ may happen due to reproduction but $\theta_{2} \neq \theta_{1}$. Suppose $\mathrm{P}$ and $\mathrm{Q}$ represent the respective positions of the two bacteria as shown in Figure 1). At the start of reproduction, $\theta_{1}$ and $\theta_{2}$ remain apart from each other but as the process progresses they come close to each other gradually.

\subsection{Analytical Treatment}

In our two bacterial system $\theta_{1}(t)$ and $\theta_{2}(t)$ represent the position of the two bacteria at time $t$ and $J\left(\theta_{1}\right), J\left(\theta_{2}\right)$ denote the cost function values at those positions respectively. The accumulated cost may be mathematically 
modeled as $\int_{0}^{t} J\left(\theta_{1}(t)\right) d t$. For a minimization problem,

higher accumulated cost represents that a bacterium did not get as many nutrients during its lifetime of foraging and hence is not as "healthy" and thus unlikely to reproduce. The two-bacterial system working on a single-dimensional fitness landscape is depicted in Figure 1.

To simulate the bacterial reproduction we have to take a decision on which bacterium will split in next generation and which one will die. This decision may be modeled with the help of the well-known unit step function $u(x)$ (also known as Heaviside step function [15]), which is defined as,

$$
\begin{aligned}
u(x) & =1 \text {; If } x>0 \\
& =0 \text {; if } x<0
\end{aligned}
$$

In what follows, we shall denote $\theta_{1}(t)$ and $\theta_{2}(t)$ as $\theta_{1}$ and $\theta_{2}$ respectively. Now if we consider that $\Delta \theta_{1}$ is the infinitesimal displacement $\left(\Delta \theta_{1} \rightarrow 0\right)$ of the first bacterium in infinitesimal time $\Delta t(\Delta t \rightarrow 0)$ towards the second bacterium in favorable condition i.e. when the second is healthier than the first one, then the instantaneous velocity of the first one is given by, $\frac{\Delta \theta_{1}}{\Delta t}$. How this bacterial movement takes place is shown in figure. 2. Now when we are trying to model reproduction we assume the instantaneous velocity of the worse bacterium to be proportional with the distance between the two bacteria, i.e. as they come closer their velocity decreases but this occurs unless we incorporate the decision making part. So, if the first bacterium is the worse one then,

$$
\begin{aligned}
& \quad \frac{\Delta \theta_{1}}{\Delta t} \infty\left(\theta_{2}-\theta_{1}\right) \\
& \Rightarrow \frac{\Delta \theta_{1}}{\Delta t}=\bar{k}\left(\theta_{2}-\theta_{1}\right) \quad \text { [Where, } \bar{k} \text { is the } \\
& \text { proportionality constant] } \\
& \Rightarrow \frac{\Delta \theta_{1}}{\Delta t}=1 .\left(\theta_{2}-\theta_{1}\right)=\left(\theta_{2}-\theta_{1}\right)
\end{aligned}
$$

[If we assume that $\bar{k}=1 \sec ^{-1}$ ]

Then we may model the decision making part with the unit step function in the following way:

$$
\frac{\Delta \theta_{1}}{\Delta t}=u\left[\int_{0}^{t} J\left(\theta_{1}\right) d t-\int_{0}^{t} J\left(\theta_{1}+\Delta \theta_{1}\right) d t\right] .\left(\theta_{2}-\theta_{1}\right)
$$

Similarly, when we consider the second bacterium, we get,

$$
\frac{\Delta \theta_{2}}{\Delta t}=u\left[\int_{0}^{t} J\left(\theta_{2}\right) d t-\int_{0}^{t} J\left(\theta_{2}+\Delta \theta_{2}\right) d t\right] .\left(\theta_{1}-\theta_{2}\right)
$$

In equation (5), $\int_{0}^{t} J\left(\theta_{1}\right) d t$ represents the health of the first bacterium at the time instant $t$ and $\int_{0}^{t} J\left(\theta_{1}+\Delta \theta_{1}\right) d t$ represents the health corresponding to $\left(\theta_{1}+\Delta \theta_{1}\right)$ at the time instant $t$.
We are going to carry out calculations with the equation for bacterium 1 only, as the results for other bacterium can be obtained in a similar fashion.

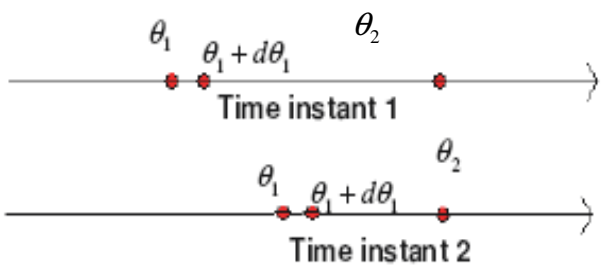

Fig 1. Change of position of the bacteria during reproduction.

We write the equation (5) corresponding to bacterium 1 as,

$\Rightarrow \frac{\Delta \theta_{1}}{\Delta t}=u\left[-\int_{0}^{t} \frac{J\left(\theta_{1}+\Delta \theta_{1}\right)-J\left(\theta_{1}\right)}{\Delta t} d t\right]\left(\theta_{2}-\theta_{1}\right)$

$[\because \Delta t>0$. We know for a positive constant $\Delta t$,

$u\left(\frac{x}{\Delta t}\right)=u(x)$ as $x$ and $\frac{x}{\Delta t}$ are of same sign and unit

step function depends only upon sign of the argument. ]

$\Rightarrow \underset{\substack{\Delta t \rightarrow 0 \\ \Delta \theta_{1} \rightarrow 0}}{L t} \frac{\Delta \theta_{1}}{\Delta t}=\underset{\substack{\Delta t \rightarrow 0 \\ \Delta \theta_{1} \rightarrow 0}}{L t} u\left[-\int_{0}^{t} \frac{J\left(\theta_{1}+\Delta \theta_{1}\right)-J\left(\theta_{1}\right)}{\Delta \theta_{1}} \frac{\Delta \theta_{1}}{\Delta t} d t\right] .\left(\theta_{2}-\theta_{1}\right)$

Again, $J(x)$ is assumed to be continuous and differentiable.

$\operatorname{Lim}_{\Delta \theta \rightarrow 0} \frac{J\left(\theta_{1}+\Delta \theta_{1}\right)-J\left(\theta_{1}\right)}{\Delta \theta_{1}}$ is the value of the gradient at that point and may be denoted by $\frac{d J\left(\theta_{1}\right)}{d \theta_{1}}$ or $G_{1}$. So we write,

$\Rightarrow \frac{d \theta_{1}}{d t}=u\left[-\int_{0}^{t}\left(\frac{d J}{d \theta_{1}} \frac{d \theta_{1}}{d t}\right) d t\right] .\left(\theta_{2}-\theta_{1}\right)\left[\right.$ Where $\frac{d \theta_{1}}{d t}$ is

the instantaneous velocity of the first bacterium]

$\Rightarrow v_{1}=u\left[-\int_{0}^{t} G_{1} v_{1} d t\right] .\left(\theta_{2}-\theta_{1}\right)$

[where $v_{1}=\frac{d \theta_{1}}{d t}$ and $G_{1}$ is the gradient of $J$ at $\theta=\theta_{1}$.]

Now in equation (5) we have not yet considered the fact that the event of reproduction is taking place at $t=1$ only. So we must introduce a function of time $r(t)=2 * u\left(-(t-1)^{2}\right)$ (unit step) $\quad\left(u\left(-(t-1)^{2}\right)\right.$ is multiplied with 2 for getting $r(t)=1$, not 0.5 , when $t=1)$ in product with the right hand side of equation (5). This provides a sharp impulse of strength 1 unit at time $t=1$. Now it is well known that $u(x)$ may be approximated with the continuous logistic function $\phi(x)$, where $\phi(x)=\frac{1}{1+e^{-k x}}$. 
We note that,

$$
u(x)=\operatorname{Lt}_{k \rightarrow \infty} \phi(x)=\operatorname{Lt}_{k \rightarrow \infty} \frac{1}{1+e^{-k x}}
$$

Figure 2 illustrates how the logistic function may be used to approximate the unit step function used for decision-making in reproduction.
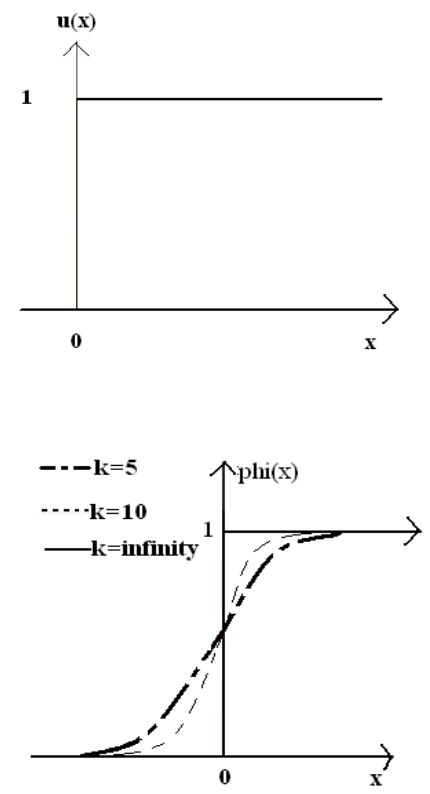

Fig. 2. The unit step and the logistic functions

Following this we may write:

$$
r(t)=2 * u\left(-(t-1)^{2}\right) \approx \frac{2}{1+e^{k(t-1)^{2}}}
$$

For moderately large value of $k$, since $t \rightarrow 1$, we can have $\left|k(t-1)^{2}\right|<<1$ and thus $e^{k(t-1)^{2}} \approx 1+k(t-1)^{2}$. Using this approximation of the exponential term we may replace the unit step function $r(t)$ with another continuous function $g(\mathrm{t})$ where

$$
g(t)=\frac{2}{2+k(t-1)^{2}},
$$

which is not an impulsive function just at $t=1$ rather a continuous function as shown in Figure 3. Higher value of $k$ will produce more effective result. Due to the presence of this function we see that

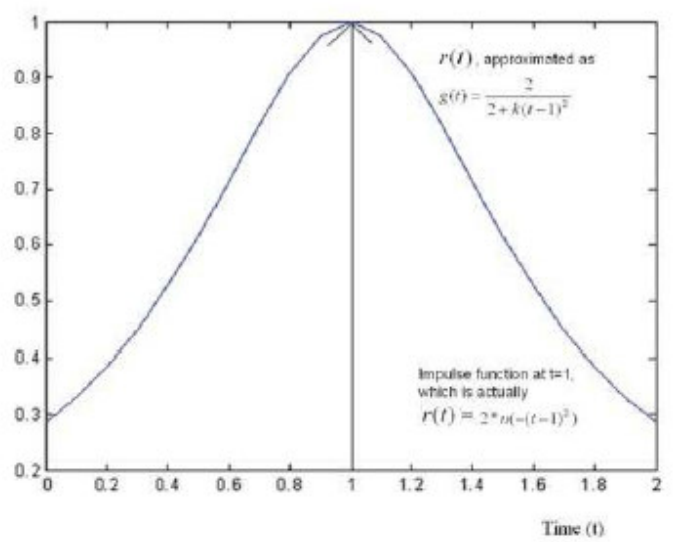

Fig 3. Function $r(t)$ and $g(t)$

$v_{1}\left(i . e, \frac{d \theta_{1}}{d t}\right)$ will be maximum at $\mathrm{t}=1$ and decreases drastically when we move away from $\mathrm{t}=1$ in both sides.

So equation (7) is modified and becomes,

$$
v_{1}=u\left[-\int_{0}^{t} G_{1} v_{1} d t\right]\left(\theta_{2}-\theta_{1}\right) \cdot \frac{2}{2+k(t-1)^{2}}
$$

For ease of calculation we denote the term within the unit step function as $M=-\int_{0}^{t} G_{1} v_{1} d t$ to obtain,

$$
\begin{gathered}
v_{1}=u(M)\left(\theta_{2}-\theta_{1}\right) \cdot \frac{2}{2+k(t-1)^{2}} \\
\text { Since } u(M)=\operatorname{Lt}_{\alpha \rightarrow \infty} \frac{1}{1+e^{-\alpha M}}
\end{gathered}
$$

We take a smaller value of $\alpha$ for getting into the mathematical analysis (say $\alpha=10$ ). Since, we have the region, under consideration with very low gradient and the velocity of the particle is low, (so product $G_{1} v_{1}$ is also small enough), and the time interval of the integration is not too large (as the time domain under consideration is not large), so it is possible to write, by expanding the exponential part and neglecting the higher order terms

$$
\begin{aligned}
u(M) & =\frac{1}{1+(1-\alpha M)} \\
& =\frac{1}{2(1-\alpha M / 2)}
\end{aligned}
$$

Substituting this expression in equation (10) we get,

$$
\begin{aligned}
& v_{1}=\frac{1}{2(1-\alpha M / 2)}\left(\theta_{2}-\theta_{1}\right) \frac{2}{2\left(1+(k / 2)(t-1)^{2}\right)} \\
\Rightarrow & \frac{v_{1}}{\theta_{2}-\theta_{1}}\left(\left(1+(k / 2)(t-1)^{2}\right)=\frac{1}{2}\left(1+\frac{\alpha M}{2}\right)\right.
\end{aligned}
$$


$\left[\because\left|\theta_{2}-\theta_{1}\right| \rightarrow 0\right.$ but $\left|\theta_{2}-\theta_{1}\right| \neq 0$ also $\because\left|\frac{\alpha M}{2}\right| \ll<1$, neglecting higher order

terms, $\left.\quad\left(1-\frac{C M}{2}\right)^{-1} \approx\left(1+\frac{C M}{2}\right)\right]$

Now the equation given by (11) is true for all values possible values of $t$, so we can differentiate both sides of it with respect to $\mathrm{t}$ and get,

$$
\begin{aligned}
& \Rightarrow \frac{\left(\theta_{2}-\theta_{1}\right) \frac{d v_{1}}{d t}-v_{1}\left(\frac{d \theta_{2}}{d t}-\frac{d \theta_{1}}{d t}\right)}{\left(\theta_{2}-\theta_{1}\right)^{2}}\left(\left(1+(k / 2)(t-1)^{2}\right)\right. \\
& +\frac{v_{1}}{\theta_{2}-\theta_{1}} k(t-1)=\frac{1}{4} \frac{d(C M)}{d t} \\
& \text { Now, } \frac{d(C M)}{d t}=\frac{d\left(-\alpha \int_{0}^{t} v_{1} G_{1} d t\right)}{d t}=-\alpha v_{1} G_{1}
\end{aligned}
$$

[By putting the expression for $\mathrm{M}$ and applying the Leibniz theorem for differentiating integrals] So from (12), we get,

$$
\begin{aligned}
& \frac{\left(\theta_{2}-\theta_{1}\right) \frac{d v_{1}}{d t}-v_{1}\left(\frac{d \theta_{2}}{d t}-\frac{d \theta_{1}}{d t}\right)}{\left(\theta_{2}-\theta_{1}\right)^{2}}\left(\left(1+(k / 2)(t-1)^{2}\right)\right. \\
& +\frac{v_{1}}{\theta_{2}-\theta_{1}} k(t-1)=-\frac{1}{4} \alpha v_{1} G_{1}
\end{aligned}
$$

Putting $\frac{d \theta_{1}}{d t}=v_{1}$ and $\frac{d \theta_{2}}{d t}=v_{2}$ after some further manipulations (where we need to cancel out $\left(\theta_{2}-\theta_{1}\right)$, which we can do as $\left|\theta_{2}-\theta_{1}\right| \rightarrow 0$ towards the end of reproduction but never $\left|\theta_{2}-\theta_{1}\right| \neq 0$ according to assumption (iii)), we get,

$$
\begin{aligned}
& \frac{d v_{1}}{d t}=-\frac{v_{1}^{2}}{\theta_{2}-\theta_{1}}-v_{1}\left[\frac{k(t-1)}{1+(k / 2)(t-1)^{2}}+\right. \\
& \left.\frac{\alpha G_{1}\left(\theta_{2}-\theta_{1}\right)}{4\left(1+(k / 2)(t-1)^{2}\right)}-\frac{v_{2}}{\theta_{2}-\theta_{1}}\right] \\
& \Rightarrow \frac{d v_{1}}{d t}=-P v_{1}^{2}-Q v_{1}
\end{aligned}
$$

where, $P=\frac{1}{\theta_{2}-\theta_{1}}$ and

$$
\begin{aligned}
& Q=\left(\frac{k(t-1)}{1+(k / 2)(t-1)^{2}}+\frac{\alpha G_{1}\left(\theta_{2}-\theta_{1}\right)}{4\left(1+(k / 2)(t-1)^{2}\right)}\right. \\
& \left.-\frac{v_{2}}{\theta_{2}-\theta_{1}}\right)
\end{aligned}
$$

\section{STABILITY ANALYSIS}

Now for gaining further mathematical insight we do some simplifications over equation (13). The effect of reproduction is mostly pronounced around $t=1$, so $(t-1) \rightarrow 0$. Thus we can neglect the first expression in $Q$, which contains $(t-1)$. Again we restrict our analysis to regions only where gradient is very low, i.e., $G_{1} \rightarrow 0$. So we can also neglect the second expression in $Q$, which contains $G_{1}$. Thus we get a simplified version of the acceleration of the first bacterium as,

$$
\begin{aligned}
& \frac{d v_{1}}{d t}=-\frac{v_{1}^{2}}{\theta_{2}-\theta_{1}}+\frac{v_{1} v_{2}}{\theta_{2}-\theta_{1}} \\
& \Rightarrow \frac{d^{2} \theta_{1}}{d t^{2}}=\frac{\frac{d \theta_{1}}{d t}\left(v_{2}-\frac{d \theta_{1}}{d t}\right)}{\left(\theta_{2}-\theta_{1}\right)}
\end{aligned}
$$

Now we will be undertaking a state variable analysis, Let us assume, that $x_{1}$ and $x_{2}$ are two state variables (velocity and position of the second bacterium are assumed to remain unchanged during this analysis), where

$$
x_{1}=\theta_{1} \text { and } x_{2}=\frac{d \theta_{1}}{d t}
$$

So we get,

$$
\begin{aligned}
& \dot{x_{1}}=x_{2}=f_{1}\left(x_{1}, x_{2}\right) \\
& \dot{x_{2}}=\frac{x_{2}\left(v_{2}-x_{2}\right)}{\left(\theta_{2}-x_{1}\right)}=f_{2}\left(x_{1}, x_{2}\right)
\end{aligned}
$$

This is a nonlinear system and we want to perform the stability analysis of the system in a small region (Figure 4) around the equilibrium point. Let $\overrightarrow{x_{e}}=\left[x_{1}^{*}, x_{2}^{*}\right]$ be the equilibrium point of the system. So we have $f_{1}\left(x_{1}^{*}, x_{2}^{*}\right)=f_{2}\left(x_{1}^{*}, x_{2}^{*}\right)=0$. Now,

$$
\begin{aligned}
& \frac{d x_{1}}{d t}=f_{1}\left(x_{1}, x_{2}\right)=f_{1}\left(x_{1}^{*}, x_{2}^{*}\right)+\left.\frac{\partial f_{1}}{\partial x_{1}}\right|_{\substack{x_{1}=x_{1}^{*} \\
x_{2}=x_{2}}}\left(x_{1}-x_{1}^{*}\right) \\
& +\left.\frac{\partial f_{1}}{\partial x_{2}}\right|_{\substack{x_{1}=x_{1}^{*} \\
x_{2}=x_{2}}}\left(x_{2}-x_{2}^{*}\right)
\end{aligned}
$$

[By expanding with Taylors series around the equilibrium point] 


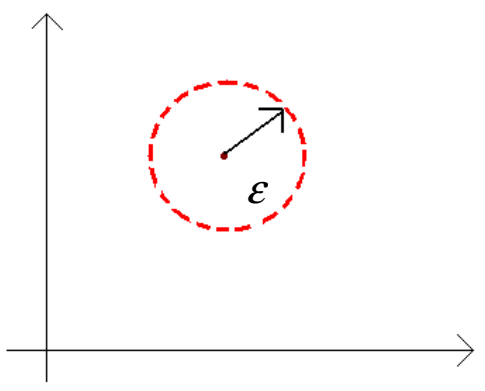

Fig 4. The region in which our stability analysis is valid.

\[ \frac{d x_{2}}{d t}=f_{2}\left(x_{1}, x_{2}\right)=f_{2}\left(x_{1}^{*}, x_{2}^{*}\right)+ \]
Similarly, $\left.\frac{\partial f_{2}}{\partial x_{1}}\right|_{\substack{x_{1}=x_{1}^{*} \\ x_{2}=x_{2}^{*}}}\left(x_{1}-x_{1}^{*}\right)+\left.\frac{\partial f_{2}}{\partial x_{2}}\right|_{\substack{x_{1}=x_{1}^{*} \\ x_{2}=x_{2}^{*}}}\left(x_{2}-x_{2}^{*}\right)$

Let, $p=x_{1}-x_{1}^{*}$ and $q=x_{2}-x_{2}^{*}$

$\Rightarrow \frac{d p}{d t}=\frac{d x_{1}}{d t}$ and $\frac{d q}{d t}=\frac{d x_{2}}{d t}$

From (16),

$$
\begin{aligned}
& \frac{d p}{d t}=\left.\frac{\partial f_{1}}{\partial x_{1}}\right|_{\substack{x_{1}=x_{1}^{*} \\
x_{2}=x_{2}^{*}}} p+\left.\frac{\partial f_{1}}{\partial x_{2}}\right|_{\substack{x_{1}=x_{1}^{*} \\
x_{2}=x_{2}}} q \\
& {\left[\because f_{1}\left(x_{1}^{*}, x_{2}^{*}\right)=f_{2}\left(x_{1}^{*}, x_{2}^{*}\right)=0\right]} \\
& \frac{d q}{d t}=\left.\frac{\partial f_{2}}{\partial x_{1}}\right|_{\substack{x_{1}=x_{1}^{*} \\
x_{2}=x_{2}^{*}}} p+\left.\frac{\partial f_{2}}{\partial x_{2}}\right|_{\substack{x_{1}=x_{1}^{*} \\
x_{2}=x_{2}^{*}}} q \\
& {\left[\because f_{1}\left(x_{1}^{*}, x_{2}^{*}\right)=f_{2}\left(x_{1}^{*}, x_{2}^{*}\right)=0\right]}
\end{aligned}
$$

Writing the above equations in a more compact form we get,

$$
\begin{gathered}
{\left[\begin{array}{c}
\dot{p} \\
\dot{q}
\end{array}\right] \nabla_{1}\left[\begin{array}{ll}
\frac{\partial f_{1}}{d x_{1}} & \frac{\partial f_{1}}{d x_{2}} \\
\frac{\partial f_{2}}{d x_{1}} & \frac{\partial f_{2}}{d x_{2}}
\end{array}\right]_{\vec{x}=\vec{x}_{e}}\left[\begin{array}{l}
p \\
q
\end{array}\right]} \\
\text { where } J=\left[\begin{array}{ll}
\frac{\partial f_{1}}{d x_{1}} & \frac{\partial f_{1}}{d x_{2}} \\
\frac{\partial f_{2}}{d x_{1}} & \frac{\partial f_{2}}{d x_{2}}
\end{array}\right]_{\vec{x}=\vec{x}_{e}}
\end{gathered}
$$

is the Jacobian matrix. If $f_{1}$ and $f_{2}$ are given by equations (14) and (15), Then equation (18) becomes as,

$$
\left[\begin{array}{l}
\dot{p} \\
\dot{q}
\end{array}\right]=\left[\begin{array}{cc}
0 & 1 \\
\frac{x_{2}^{*}\left(v_{2}-x_{2}^{*}\right)}{\left(\theta_{2}-x_{1}^{*}\right)^{2}} & \frac{\left(v_{2}-2 x_{2}^{*}\right)}{\left(\theta_{2}-x_{1}^{*}\right)}
\end{array}\right]\left[\begin{array}{l}
p \\
q
\end{array}\right]
$$

which is a linear state space representation of the nonlinear reproduction system around the equilibrium point.

Let, $M=\frac{x_{2}^{*}\left(v_{2}-x_{2}^{*}\right)}{\left(\theta_{2}-x_{1}^{*}\right)^{2}}$ and $\quad N=\frac{\left(v_{2}-2 x_{2}^{*}\right)}{\left(\theta_{2}-x_{1}^{*}\right)}$, then $\mathbf{J}$ matrix looks like,

$$
J=\left[\begin{array}{cc}
0 & 1 \\
M & N
\end{array}\right]
$$

Both of the eigen values of this matrix are real and they are,

$$
\lambda_{1}=\frac{-2 x_{2}^{*}}{\left(\theta_{2}-x_{1}^{*}\right)} \text { and } \lambda_{2}=\frac{2\left(v_{2}-x_{2}^{*}\right)}{\left(\theta_{2}-x_{1}^{*}\right)} \text {. }
$$

Now we are to determine when $f_{1}\left(x_{1}, x_{2}\right)=f_{2}\left(x_{1}, x_{2}\right)=0$, i.e., equilibrium values of the two state variables.

We find that at $x_{2}^{*}=0$ is a solution at which the system is in equilibrium, as then the rate of change of both the state variables becomes zero.

When, $x_{2}^{*}=0, \lambda_{1}=0$ and $\lambda_{2}=\frac{2 v_{2}}{\left(\theta_{2}-x_{1}^{*}\right)}$

For $\lambda_{2}<0$, (for system stability it is urgently required), we have two options,

Either $\left(v_{2}<0\right.$ and $\left.\theta_{2}>x_{1}^{*}\right)$ or $\left(v_{2}>0\right.$ and $\left.\theta_{2}<x_{1}^{*}\right)$ But in any case $\lambda_{1}$ is zero, which ensures a constant component of the state variables even in the final stage. So to assure full stability, we must have

Situation 1: $\left(x_{2}^{*}=+\mathcal{E}, v_{2}<0\right.$, and $\left.\theta_{2}>x_{1}^{*}\right)$ or

Situation 2: $\left(x_{2}^{*}=-\varepsilon, v_{2}>0\right.$ and $\left.\theta_{2}<x_{1}^{*}\right)$

That means at equilibrium the first bacterium should have an infinitesimal amount of positive or negative velocity to ensure stability of this reproductive system.

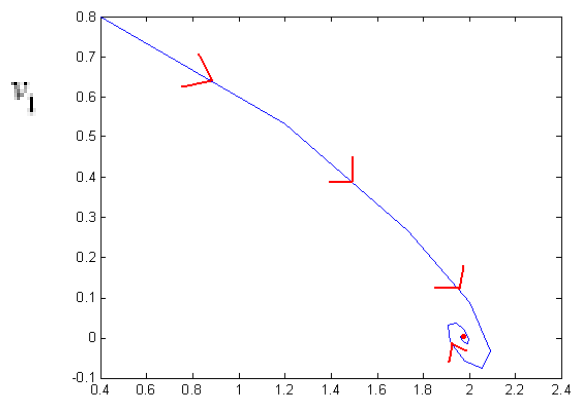

(a) 


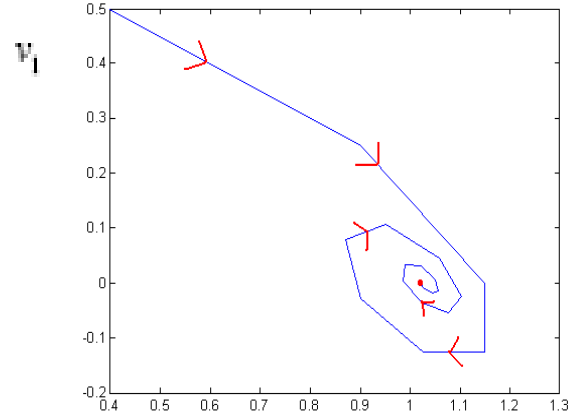

(b)

Fig 5: How the velocity and displacement of the first bacterium converge towards the equilibrium (red dot is the equilibrium point).

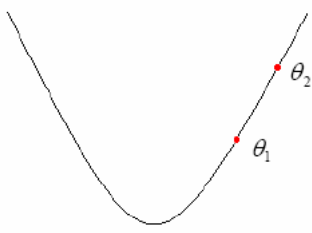

(a)

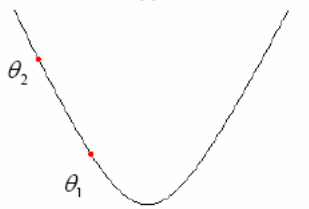

(c)

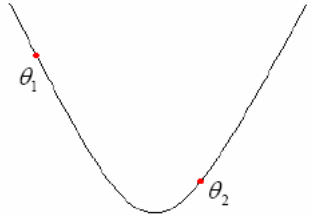

(b)

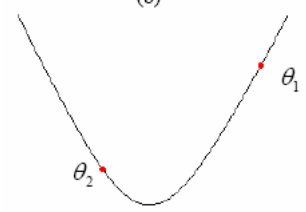

(d)
Fig 6: Probable positions of the two bacteria

Now situation 1 is true when the second bacterium is in the part of the fitness landscapes where the slope is positive and the situation 2 is valid where the second one is on negative sloped fitness landscape part [Since in the positive sloped part, velocity $v_{2}$ can never be positive and in the negative sloped part, velocity $v_{2}$ can never be negative, as BFOA is a greedy search]. Case I corresponds to situation I and, case II corresponds to situation II.

Case 1: Both bacteria are in the same slope of the fitness landscape $(6(a)$ and $6(b))$ :

In both the cases $\theta_{2}>x_{1}^{*}\left(\cong x_{1}\right)$. Now we see that in Figure 6(a) second bacterium is eventually less fit and as a result of it first bacterium never undergoes reproduction towards the other one. So the only possible case of reproduction in this scenario is $6(\mathrm{~b})$.
Case 1: Both bacteria are in the opposite slope of the fitness landscape $(6(\mathrm{c})$ and $6(\mathrm{~d}))$ :

In both the cases $\theta_{2}<x_{1}^{*}\left(\cong x_{1}\right)$. Now we see that in $6(\mathrm{c})$ second bacterium is eventually less fit and as a result of it first bacterium never undergoes reproduction towards the other one. So the only possible case of reproduction in this scenario is $6(\mathrm{~d})$.

So for stable and effective reproductive system bacterium must lie on fitness landscapes as shown in Figures 6(b) and $6(d)$

\section{CONCLUSIONS}

This paper presented a mathematical analysis of the reproduction operator of the bacterial foraging optimization algorithm. First the reproduction step dynamics is modeled and then it is represented in a state space model. Finally a stability analysis is performed on the derived model. On the basis of that analysis we try to derive some conclusions regarding the relative positions of the two bacteria in a onedimensional two bacterial system, for which a stable reproduction event can take place. Future research work may focus on deriving some control system model of the reproductive system and then further stability analysis can be undertaken.

\section{REFERENCES}

[1] Passino, K. M.: Biomimicry of Bacterial Foraging for Distributed Optimization and Control, IEEE Control Systems Magazine, 52-67, (2002).

[2] Liu, Y. and Passino, K. M.: Biomimicry of Social Foraging Bacteria for Distributed Optimization: Models, Principles, and Emergent Behaviors, Journal of Optimization Theory And Applications: Vol. 115, No. 3, pp. 603-628, December 2002.

[3] Kim, D. H., Abraham, A., Cho, J. H.: A hybrid genetic algorithm and bacterial foraging approach for global optimization, Information Sciences, Vol. 177 (18), 3918-3937, (2007).

[4] Mishra, S.: A hybrid least square-fuzzy bacterial foraging strategy for harmonic estimation. IEEE Trans. on Evolutionary Computation, vol. 9(1): 61-73, (2005).

[5] Tripathy, M., Mishra, S., Lai, L. L. and Zhang, Q. P.: Transmission Loss Reduction Based on FACTS and Bacteria Foraging Algorithm. PPSN, 222-231, (2006).

[6] Kim, D. H., Cho, C. H.: Bacterial Foraging Based Neural Network Fuzzy Learning. IICAI 2005, 20302036.

[7] Mishra, S. and Bhende C. N.: Bacterial Foraging Technique-Based Optimized Active Power Filter for Load Compensation, IEEE Transactions on Power Delivery, Volume 22, Issue 1, Jan. 2007 Page(s): 457 465.

[8] Tripathy, M. and Mishra, S.: Bacteria Foraging-Based to Optimize Both Real Power Loss and Voltage Stability Limit, IEEE Transactions on Power Systems, Vol. 22(1), 240-248, (2007). 
[9] Bonabeau, E., Dorigo, M. and Theraulaz, G.: Swarm Intelligence: From Natural to Artificial Systems. New York: Oxford Univ. Press, 1999.

[10] Kennedy, J., Eberhart R., and Shi, Y.: Swarm Intelligence, Morgan Kaufmann (2001).

[11] Kennedy, J and Eberhart R.: Particle swarm optimization. Proc. IEEE Int. conf. Neural Networks. (1995) 1942-1948.

[12] Dorigo, M. and Stiizle, T.: Ant colony optimization, MIT Press, Cambridge, MA, (2004).

[13] Back, T., Fogel, D. B. and Michalewicz, Z., Handbook of Evolutionary Computation, IOP and Oxford University Press, Bristol, UK, 1997.

[14] Abraham, A., Biswas, A., Dasgupta, S., and Das, S., Analysis of Reproduction Operator in Bacterial Foraging Optimization, IEEE Congress on Evolutionary Computation CEC 2008, IEEE World
Congress on Computational Intelligence, WCCI 2008, IEEE Press, USA, pp. 1476-1483, 2008.

[15] Anwal, R. P.: Generalized Functions: Theory and Technique, 2nd ed. Boston, MA: Birkhãuser, (1998).

[16] Biswas, A., Dasgupta, S., Das, S., and Abraham, A., Synergy of Differential Evolution and Bacterial Foraging Algorithm for Global Optimization, Neural Network World, Volume 17, No. 6, pp. 607-626, 2007.

[17] Dasgupta, S., Biswas, A., Abraham, A. and Das, S., Adaptive Computational Chemotaxis in Bacterial Foraging Algorithm, Second International Conference on Complex, Intelligent and Software Intensive Systems, Barcelona, Spain, IEEE Computer Society Press, pp. 64-71, 2008. 